\title{
Fifty years of progress in community mental health in US: the growth of evidence-based practices
}

\author{
L. B. Dixon* and E. C. Schwarz \\ New York State Psychiatric Institute, Department of Psychiatry, College of Physicians \& Surgeons, Columbia University, New York, USA
}

Fifty years have elapsed since the passage of the Community Mental Health Centers (CMHC) Act in 1963 that reflected the legislative peak of the community mental healthcare movement in the US Progress of the last 10 years is represented both by expansions of evidence-based practices (EBPs) and the development of emerging practices and fundamental shifts in the orientation of the system stimulated by the consumer-driven recovery movement. Established EBPs have accumulated expanded evidence, new EBPs have been developed and emerging EBPs are gaining increased acceptance. While the lack of widespread implementation of EBPs as well as the limitations of these technologies produces unnecessary suffering and disability, we believe that the growth of evidence for treatments and services justifies optimism for the future.

First published online 12 November 2013

Key words: Evidence-based practices, mental health, recovery, schizophrenia.

Fifty years have elapsed since the passage of the Community Mental Health Centers (CMHC) Act in 1963 that reflected the legislative peak of the community mental health movement in the US. Have we made progress in the last 10 years? Dixon \& Goldman (2003) previously reflected on this question at the 40-year anniversary of the Act, underlining the importance of the development of evidence-based practices (EBPs) in our evolving technologies to treat serious mental illness. The last decade has seen expansions in the development of EBPs. The last decade has also witnessed the development of strategies and technologies to implement these practices and to personalize their use as reflected in the development of tools for shared decision making. These advances provide the basis for optimism. We briefly summarize the history of CMHCs, update the status of EBPs in the care of individuals diagnosed with schizophrenia and discuss new developments in implementation and personalization.

\section{Historical background of Community Mental Health Centers (CMHC)}

The notion of 'cycles of reform' articulated by Morrissey \& Goldman (1984) helps explain the pathway to CMHCs and their current role in providing mental health services. The first cycle of the 19th century introduced moral treatment and asylum and

* Address for correspondence: Dr L. Dixon, New York State Psychiatric Institute, Room 2702, Box 100, 1051 Riverside Drive, New York, NY 10032, USA.

(Email: DixonLi@nyspi.columbia.edu) merged into the second cycle of the early 20th century that featured mental hygiene and the introduction of the psychopathic hospital. These cycles began with optimism and the public's support for a new and innovative approach to mental healthcare but ultimately gave way to some disappointment. Although the environmental improvements helped some individuals with acute and less severe forms of illness, they did not produce better outcomes or cures for individuals with severe mental illnesses. The third cycle of reform of the mid-20th century spawned the community mental health center (CMHC) and the CMHC Act. The hope of this cycle was the healing capacity of the community as an antidote for the toxicity of institutions. Propelled by the advent of antipsychotic and antidepressant medications, the population of state hospitals declined from 560000 to 140000 . More than $700 \mathrm{CMHCs}$ were created that served catchment areas representing more than $50 \%$ of the population. Unfortunately, the combination of inadequate and uncoordinated mental healthcare and unmet social welfare and housing needs of individuals with severe mental illnesses created the crisis that led to the fourth cycle of reform that focused on the community care of individuals who were already severely disabled by mental illness.

The Community Support Programme (CSP) embodied the federal response to the failure of deinstitutionalization. The National Institute of Mental Health (NIMH) funded 19 states to pilot three-year programmes that would provide services 'for one particularly vulnerable population - adult psychiatric patients whose disabilities are severe and persistent but for whom long-term skilled or semiskilled nursing care 
is inappropriate.' The CSP included a network of services including crisis care, psychosocial rehabilitation, medical and mental healthcare, case management and some assistance with housing and employment. This pilot programme became a model for the country. As emphasized by Goldman \& Dixon (2003) in their 40th anniversary review, the technology offered by the development of EBPs such as Assertive Community Treatment (ACT), has been critical to the success of CSP.

\section{Progress in the last decade}

The last decade has perhaps represented the tail end of the fourth cycle, while the Affordable Care Act and changes in healthcare financing and delivery portend a new cycle of reform with its attendant optimism. Anticipation of the next cycle and the promise of the future could cause us to focus on the current problems that require a remedy - the persistent under-treatment of mental illnesses and access to mental healthcare services for many who lack insurance, the disparities in meeting the somatic healthcare needs of persons with serious mental illness and the extent to which the US lags behind other nations in developing early intervention programmes for psychosis. However, awareness of such deficits should not diminish recognition of the accomplishments of the last decade. This progress is represented both by expansions of EBPs and the development of emerging practices and fundamental shifts in the orientation of the system stimulated by the consumer-driven recovery movement.

While the evidence base has expanded for multiple psychiatric disorders, for the purposes of this editorial, we focus here on schizophrenia and the third and most recent review of psychosocial EBPs for treatment of individuals with schizophrenia conducted by the Schizophrenia Patient Outcomes Research Team (PORT) (Dixon et al. 2010; Kreyenbuhl et al. 2010). As this review was published in 2010, it synthesizes research conducted in the five prior years and research that was not previously reviewed by the PORT team. The PORT team lists eight treatment recommendations, of which six are extensions of recommendations from past PORT reviews, e.g., ACT, supported employment (SE), family-based services, skills training, cognitive behavioural therapy, token economy interventions and two are recommendations in new areas, e.g., psychosocial treatment for co-morbid substance use disorders (SUDs) and psychosocial interventions for weight management. For illustrative purposes, we discuss the new recommendations as well as ACT, SE and family-based services that have the deepest penetration in current systems of care. Cognitive remediation, peer-based services and early intervention programmes did not meet the
PORT standard for a recommendation at that time, but have accrued a promising evidence base that many think have met the standard for EBP.

\section{Assertive community treatment}

The review of EBPs for schizophrenia begins with $\mathrm{ACT}$, perhaps the oldest and best established EBP in the US ACT goes back more than 30 years, but still engenders some controversy in the field. ACT remains a recommended EBP for individuals diagnosed with schizophrenia who are at risk for hospitalization or homelessness (Dixon et al. 2010).

Research has also extended ACT to other subpopulations and clinical outcomes, including employment, substance use and forensic populations (Dixon et al. 2010). In some cases, this has involved adding a specialized focus to ACT teams, such as SE workers and clinicians who are trained to deal with individuals who are diagnosed with co-occurring SUDs. With respect to employment within the ACT, studies vary in terms of their inclusion of employment specialists, the primary research questions being asked and the nature of the comparison condition. That considered, studies found that individuals randomized to the ACT condition were significantly more likely to have had paid employment (Chandler et al. 1997), to be working in competitive employment (McFarlane et al. 2000; Resnick et al. 2003) and to have worked in the previous 6 months relative to those in the standard care condition (Chandler et al. 1999). Furthermore, individuals in the ACT condition were rated as performing more effectively in their work role (Jerrell, 1999) and were employed for longer periods of time (McFarlane et al. 2000) relative to individuals in the standard care condition.

Controversies around ACT include its association with potentially coercive practices that foster dependence rather than independence and recovery. Research originally suggested that service users who benefited from ACT would require it forever. Now we know better that ACT can be a time-limited service. (Rosenheck \& Dennis, 2001; Hackman \& Stowell, 2009). The success of ACT should not deter us from developing other strategies that are less expensive and potentially less regressive. At the same time, recent research that affirms and attempts to extend ACT's benefits affirms its place in the technologies of treatment that enhance the well-being of individuals who have serious mental illness with established disabilities.

\section{Supported employment}

Service users tell us that they would like to work and participate in competitive employment, just like their 
neighbours. For years, people with schizophrenia were told that work was too stressful. Now we know better. SE continues to accrue an evidence base for its effectiveness in helping individuals with schizophrenia achieve competitive employment. Individual Placement and Support (IPS), the most widely researched model of SE, includes individually tailored job development, rapid job search, the availability of ongoing job supports and the integration of vocational and mental health services (Dixon et al. 2010). Studies have shown that individuals receiving these services do not exhibit stress or exacerbated symptoms, contrary to common beliefs. Randomized controlled trials have consistently demonstrated the effectiveness of SE in helping persons with schizophrenia to achieve competitive employment, work more hours and earn more wages than persons who did not receive SE. In most of these studies, among those who received integrated SE and psychiatric services, $50 \%$ or more persons obtained competitive employment at some point during the study follow-up period. Outcomes relating to the amount of hours worked and wages earned were also found to be superior among those receiving $\mathrm{SE}$ in comparison to those receiving traditional vocational services. In order to boost the effect of SE, recent work has focused on augmenting vocational services with interventions such as cognitive remediation, (Wexler \& Bell, 2005; McGurk et al. 2007), social skills training (Wallace \& Tauber, 2004; Mueser et al. 2005), and cognitive behaviour therapy (Lysaker et al. 2005; Vauth et al. 2005). Each of these interventions was created as a stand-alone service. We are now learning how to put them together effectively.

We are far from claiming victory in achieving full social and economic integration of individuals with schizophrenia in our society. Many impediments to a working life remain, not the least of which is our system of tying eligibility for public health insurance to the designation of disability. At the same time, the development of IPS as an EBP represents a substantial advancement in our technology.

\section{Family-based services}

Family support has consistently been shown to be associated with better outcomes for individuals diagnosed with serious mental illness. The history of psychiatry's treatment of families raises many emotional issues for different stakeholders; family members tell stories of not only being excluded from treatment, but also of being blamed for the development of mental illness in their loved one. At the same time, consumers and families alike want their choices and preferences honoured. All the PORT reviews have recommended that persons with schizophrenia who have ongoing contact with their families, including relatives and significant others, should be offered a family intervention that lasts at least 6-9 months. Interventions that last 6-9 months have been found to significantly reduce rates of relapse and re-hospitalization (Dixon et al. 2010). Although not as consistently observed, research has found other benefits for patients, such as increased medication adherence, reduced psychiatric symptoms and reduced levels of perceived stress for patients. Family members have also been found to have lower levels of burden and distress and improved family relationships. Key elements of effective family interventions include illness education, crisis intervention, emotional support and training in how to cope with illness symptoms and related problems. The recent PORT review emphasized that the evidence base for family psychoeducation is much weaker when the consumer has not had a recent illness exacerbation, demonstrating a different type of progress in our science, i.e., correcting and clarifying the interpretations of the existing data.

In addition to providing greater precision regarding the value of longer interventions, the most recent PORT review also acknowledged the efficacy of family interventions that are shorter than 6 months (Dixon et al. 2010). Briefer models that provide education, training and support can improve the consumer's psychiatric symptoms, treatment adherence, functional and vocational status and satisfaction with treatment. Positive family outcomes include reduced family burden and increased satisfaction with family relationships. The creation of an evidence base for shorter interventions for family members potentially permits much greater feasibility and access to family-based services. The limited uptake of the longer family psychoeducation models also demonstrates the importance of creating evidence-based approaches that align with consumer preferences (Cohen et al. 2008).

\section{Psychosocial interventions for alcohol and SUDs}

Despite the frequent co-occurrence of SUDs and schizophrenia, the PORT had not previously reviewed these treatments and made no recommendation. By the third PORT, the evidence for the importance of substance use to the course of schizophrenia and the accumulation of data made it clear that this topic required consideration. The literature evaluates methods of delivery of SUD treatment (such as comparisons of integrated $v$. parallel treatment), and specific interventions for treating SUDs. The PORT review concluded that persons with schizophrenia and a co-morbid alcohol or drug use disorder should be offered treatment that includes motivational enhancement and behavioural strategies that focus on engagement in treatment, 
coping skills training, relapse prevention training and its delivery in a service model that is integrated with mental healthcare (Dixon et al. 2010).

In spite of the emerging technology in the treatment of co-occurring SUDs, far more needs to be done. The good news is that we now have a roadmap for what needs to be done, starting with the elimination of barriers dividing substance use from mental healthcare services, inhibiting treatment of the whole person.

\section{Psychosocial interventions for weight management}

The final recommendation to be discussed addresses the challenge of obesity, common among individuals diagnosed with serious mental illness, and contributing to the elevated mortality rates. Psychotropic medications and their side effects contribute to weight gain, so the notion that the treatments we provide make people less healthy underscores the importance of tackling this challenge. The PORT found that individuals with schizophrenia who are overweight (Body Mass Index 25.0-29.9) or obese (Body Mass Index greater than or equal to 30.0) should be offered a psychosocial weight loss intervention that is at least 3 months in duration to promote weight loss (Dixon et al. 2010). The key elements of psychosocial interventions for weight loss include psychoeducation focused on nutritional counselling, caloric expenditure and portion control; behavioural self-management including motivational enhancement; goal setting; regular weigh-ins; self-monitoring of daily food and activity levels; and dietary and physical activity modifications. These programmes do not produce dramatic weight loss, and no recommendation was made on how to prevent weight gain. Hence, here again we are faced with the challenge of whether to consider the cup half full or half empty. The perspective of half full remembers that the technologies of EBPs are always evolving and evidence accumulates (Daumit et al. 2013). We can hope that the technology will improve with more research and practice.

\section{Emerging evidence to recommendations}

There are at least three treatments or strategies that have a growing database and which many already consider to be EBPs. They are important to mention because they demonstrate the ongoing expansion of knowledge and also because the designation of ' $\mathrm{EBP}^{\prime}$ status is an inexact science. First, it is well established that cognitive impairment in schizophrenia is common and accounts for significant variation in real-world community outcomes such as work performance. The class of behavioural treatments known as 'cognitive remediation' specifically targets memory, attention, reasoning and similar capacities, with the ultimate aim of enhancing everyday functioning. Considerable data suggests that cognitive remediation not only improves the performance on neuropsychological tests, but also may enhance psychosocial functioning (Wykes et al. 2011).

The participation of consumers in the planning, delivery and evaluation of services is increasingly recognized as essential to a recovery-oriented and person-centred system of care. Both professional and consumer organizations have participated in efforts to develop and test models of consumers as providers of different kinds of mental healthcare services (Dixon et al. 2010). This has entailed a range of approaches including consumers serving as members of regular clinical teams, consumers providing speciality peer-to-peer services and independent consumer-run agencies (Dixon et al. 2010). Consumers can play a unique role by sharing lived experiences and serving as role models for one another. In each of these types of programmes, there is some evidence of specific benefit and/or no differences between professionally delivered and peer delivered services. This increasingly accepted approach would have not long ago been virtually unthinkable in mainstream mental healthcare services.

The final intervention deserving consideration as an EBP is psychosocial treatments for recent onset of schizophrenia (Lieberman et al. 2013). In some ways, this is not a new EBP, but a specific way of combining the existing practices and packaging them for young people. Already the standard of care in Australia, UK, Canada and other countries, the US is just beginning to catch up. The availability of strategies to finance early intervention services for psychosis may herald the next cycle of reform.

\section{Conclusion}

We have argued for progress in evaluating the last decade; we note that it is equally easy to argue the case for frustration. Specifically, the lack of widespread implementation of EBPs at all and in a manner that is patient-centred as well as the limitations of these technologies, produces unnecessary suffering and disability. Nevertheless, we believe that the growth of evidence for treatments and services justifies optimism for the future. Each of the previous cycles of reform floundered because of the lack of an effective treatment technology to make them succeed. We now have a methodology and a roadmap to improve these technologies, an emerging science guiding implementation and methods to measure how well we are doing. 
Acknowledgement

None.

\section{Financial Support}

This work received no specific grant from any funding agency, commercial or not from any profit sectors.

\section{Conflict of Interest}

None.

\section{Ethical Standard}

Not applicable.

\section{References}

Chandler D, Meisel J, Hu T, McGowen M, Madison K (1997). A capitated model for a cross-section of severely mentally ill clients: employment outcomes. Community Mental Health Journal 33, 501-516.

Chandler D, Spicer G, Wagner M, Hargreaves W (1999). Cost-effectiveness of a capitated assertive community treatment program. Psychiatric Rehabilitation Journal 22, 327-336.

Cohen AN, Glynn SM, Murray-Swank AB, Barrio C, Fischer EP, McCutcheon SJ, Perlick DA, Rotondi AJ, Sayers SL, Sherman MD, Dixon LB (2008). The family forum: directions for the implementation of family psychoeducation for severe mental illness. Psychiatric Services 59, 40-48.

Daumit GL, Dickerson FB, Wang NY, Dalcin A, Jerome GJ, Anderson CA, Young DR, Frick KD, Yu A, Gennusa III JV, Oefinger M, Crum RM, Charleston J, Casagrande SS, Guallar E, Goldberg RW, Campbell LM, Appel LJ (2013). A behavioral weight-loss intervention in persons with serious mental illness. New England Journal of Medicine 368, 1594-1602.

Dixon L, Goldman HH (2003). Forty years of progress in community mental health: the role of evidence-based practices. Australian and New Zealand Journal of Psychiatry 37, 668-673.

Dixon L, Dickerson F, Bellack A, Bennett M, Dickinson D, Goldberg R, Lehman A, Tenhula W, Calmes C, Pasillas R, Peer J, Kreyenbuhl J (2010). The 2009 PORT psychosocial treatment recommendations and summary statement. Schizophrenia Bulletin 36, 48-70.

Hackman AL, Stowell KR (2009). Transitioning clients from assertive community treatment to traditional mental health services. Community Mental Health Journal 45, 1-5.
Jerrell JM (1999). Skill, symptom, and satisfaction changes in three service models for people with psychiatric disability. Psychiatric Rehabilitation Journal 22, 342-348.

Kreyenbuhl J, Buchanan RW, Dickerson F, Dixon L (2010). The Schizophrenia Patient Outcomes Research Team (PORT): updated treatment recommendations 2009. Schizophrenia Bulletin 36, 94-103.

Lieberman JA, Dixon LB, Goldman HH (2013). Early detection and intervention in schizophrenia: a new therapeutic model. Journal of the American Medical Association 310, 689-690.

Lysaker PH, Bond G, Davis LW, Bryson GJ, Bell MD (2005). Enhanced cognitive-behavioral therapy for vocational rehabilitation in schizophrenia: effects on hope and work. Journal of Rehabilitation Research and Development 42, 673-682.

McFarlane WR, Dushay RA, Deakins SM, Stastny P, Lukens EP, Toran J, Link B (2000). Employment outcomes in family-aided assertive community treatment. American Journal of Orthopsychiatry 70, 203-214.

McGurk SR, Mueser KT, Feldman K, Wolfe R, Pascaris A (2007). Cognitive training for supported employment: 2-3 year outcomes of a randomized controlled trial. American Journal of Psychiatry 164, 437-441.

Morrissey JP, Goldman HH (1984). Cycles of reform in the care of the chronically mentally ill. Hospital and Community Psychiatry 35, 785-793.

Mueser KT, Aalto S, Becker DR, Ogden JS, Wolfe RS, Schiavo D, Wallace CJ, Xie H (2005). The effectiveness of skills training for improving outcomes in supported employment. Psychiatric Services 56, 1254-1260.

Resnick SG, Neale MS, Rosenheck RA (2003). Impact of public support payments, intensive psychiatric community care, and program fidelity on employment outcomes for people with severe mental illness. Journal of Nervous and Mental Disease 191, 139-144.

Rosenheck RA, Dennis D (2001). Time-limited assertive community treatment for homeless persons with severe mental illness. Archives of General Psychiatry 58, 1073-1080.

Vauth R, Corrigan PW, Clauss M, Dietl M, Dreher-Rudolph M, Stieglitz RD, Vater R (2005). Cognitive strategies versus self-management skills as adjunct to vocational rehabilitation. Schizophrenia Bulletin 31, 55-66.

Wallace CJ, Tauber R (2004). Supplementing supported employment with workplace skills training. Psychiatric Services 55, 513-515.

Wexler BE, Bell MD (2005). Cognitive remediation and vocational rehabilitation for schizophrenia. Schizophrenia Bulletin 31, 931-941.

Wykes T, Huddy V, Cellard C, McGurk SR, Czobor P (2011). A meta-analysis of cognitive remediation for schizophrenia: methodology and effect sizes. American Journal of Psychiatry 168, 472-485. 